absolute magnitude of $M_{\mathrm{B}} \approx-22.6 \mathrm{mag}$, or a lower limit for $L_{\mathrm{B}} \approx 7 L_{*}$, where $L_{*}$ is a characteristic galaxy luminosity ${ }^{33}$. Thus, OT J065349+79163 is still significantly outshining any host galaxy for the most probable host luminosity range. The properties of the $\mathrm{Mg}$ absorption lead us to expect that once the OT has faded, the galaxy could be identified optically.

Taken together, the source's compact optical appearance, a featureless continuum, X-ray emission and high redshift suggest a possible classification (independent of a burst event) as a BL Lac object $^{23}$. One of the known characteristics of BL Lac objects is their variability from radio to $\gamma$-ray wavelengths. We now evaluate the a posteriori probability that we might be seeing a BL Lac object by random coincidence with the $\gamma$-ray error box. The surface density of BL Lac objects with Rosat X-ray flux $f_{X} \leqslant 10^{-12} \mathrm{erg} \mathrm{cm}^{-2} \mathrm{~s}^{-1}$ is not very well known, but there are indications that this distribution is quite flat at low flux densities. A simple extrapolation for the expected number of BL Lacs with $f_{X}>6 \times 10^{-13} \mathrm{erg} \mathrm{cm}^{-2} \mathrm{~s}^{-1}$ is $^{24} 0.03$ per square degree. Thus the probability of finding a BL Lac object within the 3 -arcmin-radius localization region is $\sim 2 \times 10^{-4}$. The amplitude of the variability detected in the counterpart ${ }^{13}$ over a few days is also larger than has been observed in studies of BL Lac object variability ${ }^{25,26}$. Although we cannot completely exclude the possibility that OT J065349+79163 is a chance coincidence of a BL Lac object with the GRB error circle, the probability of finding a random BL Lac object which also exhibits variability that is temporally correlated with a $\gamma$-ray burst is quite small. Thus we conclude that the OT is probably associated with GRB970508, regardless of classification, though the strongest constraints naturally come from higher-energy emission.

The high redshift of OT J065349+79163, its featureless spectrum and slowly decaying optical flux are consistent with the so-called fireball models for cosmological bursts ${ }^{27-29}$, which are efficient at emitting $\gamma$-rays and produce power-law spectral energy distributions. The fluence ${ }^{30}$ of GRB970508 in the energy range 20$1,000 \mathrm{keV}$ was $3 \times 10^{-6} \mathrm{erg} \mathrm{cm}^{-2}$, and at the minimum redshift implied for OT J065349+79163, this burst would have a total $\gamma$-ray energy of $7 \times 10^{51} \mathrm{erg}$ (assuming isotropic emission). This falls in the general range of typical $\gamma$-ray burst energies from various cosmological models ${ }^{31,32}$.

The remarkable progress in detecting X-ray and optical counterparts to GRBs has been made possible only by rapid localization of the burst by BeppoSAX and prompt dissemination of the coordinates by the BeppoSAX team. Further progress in understanding GRBs requires many more optical counterparts to be identified. It is clear from experience of the first two optical counterparts that, in order to obtain the critical data, the counterparts must be discovered and followed up spectroscopically within a few days. It now seems that an understanding of the physical mechanisms behind $\gamma$ ray bursts is within reach.

Received 21 May; accepted 3 June 1997.

1. Klebesadel, R. W., Strong, I. B. \& Olsen, R. A. Observations of gamma-ray bursts of cosmic origin. Astrophys. J. 182, L85-L88 (1973).

2. Meegan, C. A. et al. Spatial distribution of gamma-ray bursts observed by BATSE. Nature 355, 143 145 (1992)

3. Lamb, D. Q. The distance scale to gamma-ray bursts. Publ. Astron. Soc. Pacif. 107, 1152-1166 (1995)

4. Paczyński, B. How far away are gamma-ray bursters? Publ. Astron. Soc. Pacif. 107, 1167-1175 (1995)

5. van Paradijs, J. et al. Transient optical emission from the error box of the $\gamma$-ray burst of 28 February 1997. Nature 386, 686-689 (1997).

6. Costa E. et al. IAU Circ. No. 6576 (1997).

7. Bond, H. E. IAU Circ. No. 6654 (1997)

8. Djorgovski, S. et al. IAU Circ. No. 6655 (1997).

9. Boella, G. et al. BeppoSAX, the wide band mission for X-ray astronomy. Astron. Astrophys. Suppl. 122, 299 (1997)

10. Costa, E. et al. IAU Circ. No. 6649 (1997)

11. Heise, J. et al. IAU Circ. No. 6654 (1997)

12. Piro, L. et al. IAU Circ. No. 6656 (1997).

13. Djorgovski, S. G. et al. The optical counterpart to the $\gamma$-ray burst GRB970508. Nature $387876-878$ (1997).

14. Oke, J. B. et al. The Keck low-resolution imaging spectrometer. Publ. Astron. Soc. Pacif. 107, 375-385 (1995).

15. Massey, P., Strobel, K., Barnes, J. V. \& Anderson, E. Spectrophotometric standards. Astrophys. J. 328 315-333 (1988).
16. Metzger, M. R. et al. IAU Circ. No. 6655 (1997).

17. Steidel, C. C. \& Sargent, W. L. W. Mg II absorption in the spectra of 103 QSOs: implications for the evolution of gas in high-redshift galaxies. Astrophys. J. Suppl. Ser. 80, 1-108 (1992).

18. Steidel, C. C., Dickinson, M. \& Persson, S. E. Field galaxy evolution since z approximately 1 from a sample of QSO absorption-selected galaxies. Astrophys. J. 437, L75-L78 (1994).

19. Steidel, C. C. in QSO Absorption Lines (ed. Meylan, G.) 139-152 (Springer, Berlin, 1995).

20. Djorgovski, S. et al. IAU Circ. No. 6660 (1997).

21. Oke, J. B. \& Korycansky, D. G. Absolute spectrophotometry of very large redshift quasars. Astrophys. J. 255, 11-19 (1982).

22. Zuo, L. \& Lu, L. Measurements of $D_{A}$ for a large QSO sample and determination of evolution of Lyman-alpha clouds. Astrophys. J. 418, 601-616 (1993).

23. Stocke, J. T. et al. The Einstein Observatory extended medium-sensitivity survey. II - The optical identifications. Astrophys. J. Suppl. Ser. 76, 813-874 (1991).

24. Nass, P. et al. BL Lacertae objects in the ROSAT All-Sky Survey: new objects and comparison of different search techniques. Astron. Astrophys. 309, 419-430 (1996).

25. Miller, H. R. \& Noble, J. C. The microvariability of blazars and related AGN. 17-29 (ASP Conf. Ser. 110, Astron. Soc. Pacif., 1996).

26. Heidt, J. \& Wagner, S. J. Statistics of optical intraday variability in a complete sample of radio-selected BL Lacertae objects. Astron. Astrophys. 305, 42-52 (1995).

27. Nészáros, P. \& Rees, M. J. Gamma-ray bursts; multiwaveband spectral predictions for blast wave models. Astrophys. J. 418, L59-L62 (1993).

28. Paczyński, B. \& Rhoads, J. E. Radio transients from gamma-ray bursters. Astrophys. J. 418, L5-L8 (1993).

29. Waxman, E. Gamma-ray burst after-glow: confirming the cosmological fireball model. Astrophys. J. (submitted).

30. Kouveliotou, C. et al. IAU Circ. No. 6660 (1997).

31. Fenimore, E. E. et al. The intrinsic luminosity of gamma-ray bursts and their host galaxies. Nature 366, 40-42 (1993).

32. Fenimore, E. E. \& Bloom, J. S. Determination of distance from time dilation of cosmological gammaray bursts. Astrophys. J. 453, 25-36 (1995).

33. Schachter, P. An analytic expression for the luminosity function of galaxies. Astrophys. J. 203, 297-306 (1976)

Acknowledgements. We thank the BeppoSAX team for their efforts in disseminating information to observers for rapid identification of an optical transient, making this work possible. We thank W. Sargent and M. Pahre for discussions, and the Keck Observatory staff for assistance at the telescope. This work is and M. Pahre for discussions, and the Keck Observatory staff for assistance at the telescope. This work is
based on observations obtained at the W. M. Keck Observatory, which is jointly operated by the California Institute of Technology and the University of California. M.R.M. was supported by Caltech; S.R.K. was supported by NASA and NSF; S.G.D. was supported by NSF and the Bressler Foundation; C.C.S. was supported by NSF and the Sloan Foundation.

Correspondence should be addressed to M.R.M. (e-mail: mrm@astro.caltech.edu).

\section{Self-trapping of incoherent white light}

\section{Matthew Mitchell \& Mordechai Segev}

Electrical Engineering Department, Center for Photonics and Optoelectronic Materials and Princeton Materials Institute, Princeton University, Princeton, New Jersey 08544, USA

Optical pulses-wave-packets-propagating in a linear medium have a natural tendency to broaden in time (dispersion) and space (diffraction). Such broadening can be eliminated in a nonlinear medium that modifies its refractive index in the presence of light in such a way that dispersion or diffraction effects are counteracted by light-induced lensing ${ }^{1,2}$. This can allow short pulses to propagate without changing their shape $\mathrm{e}^{2,3}$, and the 'self-trapping' of narrow optical beams ${ }^{1}$ whereby a beam of light induces a waveguide in the host medium and guides itself in this waveguide, thus propagating without diffraction ${ }^{4}$. Self-trapped pulses in space and time have been investigated extensively in many physical systems and, as a consequence of their particle-like behaviour, are known as 'solitons' (ref. 5). Previous studies of this phenomenon in various nonlinear media ${ }^{6-12}$ have involved coherent light, the one exception being our demonstration ${ }^{13}$ of self-trapping of an optical beam that exhibited partial spatial incoherence. Here we report the observation of self-trapping of a white-light beam from an incandescent source. Self-trapping occurs in both dimensions transverse to the beam when diffraction effects are balanced exactly by self-focusing in the host photorefractive medium. To the best of our knowledge, this is the first observation of self-trapping for any wave-packet that is both temporally and spatially incoherent. 


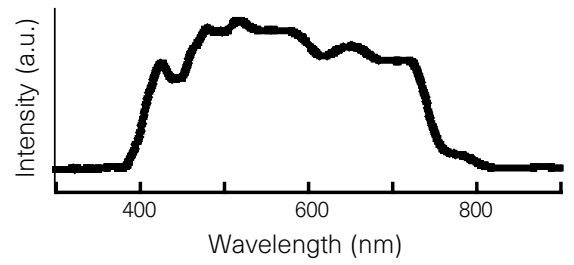

Figure 1 Spectrum of the incoherent white-light beam incident on the photorefractive crystal (a.u., arbitrary units).

The goal is to achieve self-trapping of an incoherent beam (wavepacket); that is, we wish to trap the time-averaged envelope made by a rapidly changing multi-mode broad-band optical field. At any given time, the beam contains many 'speckles': a random distribution of bright and dark patches caused by the randomly varying phase in space. To achieve self-trapping of the envelope, it is necessary to counteract the diffraction of the quickly changing speckled optical beam. The key issue is to find a nonlinear medium that responds on a timescale much longer than the rate of change of the speckle pattern of the beam. The reason for this is as follows: if an instantaneous optical nonlinearity was used, then the medium would respond to the instantaneous 'speckled' beam. Each speckle would form a small 'positive lens' and would capture a small fraction of the beam. These bright-dark features on the beam change very fast throughout propagation and these tiny inducedwaveguides intersect and cross each other in a random manner. The net effect would be beam breakup into small fragments and selftrapping of the beam's envelope would not occur. For an incoherent beam to self-trap, it is necessary that the beam induces a smooth waveguide that will guide its rapidly changing intensity at every instant. A non-instantaneous nonlinearity allows this to occur, as it reacts to a time-averaged intensity which is temporally and spatially smooth if averaged over a long enough time period. When this selfinduced waveguide guides the rapidly changing beam, self-trapping is achieved.

Photorefractive crystals are a convenient choice of such a nonlinear medium, because their response time is fully controlled by the intensity of the beam, and can be made (with low intensities) much longer than the rate of the rapid intensity fluctuations of the beam. The photorefractive self-focusing effects used here have been described in detail in conjunction with photorefractive screening solitons ${ }^{14-18}$. The formation of a bright screening soliton may be viewed in the following manner. A narrow light beam propagates in the centre of a biased dielectric (photorefractive) medium. In the illuminated region, electrons are optically excited, and therefore the conductivity increases and the resistivity decreases. Thus, the voltage drop occurs primarily in the dark regions leading to a large space-charge field $E_{\mathrm{sc}}(\mathbf{r})$ there, $\mathbf{r}$ being the coordinates in the plane transverse to the propagation direction of the beam. The change in the refractive index $\Delta n(\mathbf{r})$ is linearly proportional to the space-charge field via the electro-optic (Pockels') effect. When the polarity of the field is properly chosen, $\Delta n(\mathbf{r})$ is proportional to $-E_{\mathrm{sc}}(\mathbf{r})$ and this creates a 'graded index waveguide' that guides the beam that generated $i^{16}$.

We have recently reported the first (to our knowledge) experimental observation of self-trapping of a partially spatially incoherent optical beam ${ }^{13}$; we used a quasi-monochromatic laser beam scattered off a rotating diffuser to generate a speckled beam ${ }^{19}$ in which the phases of any two well-separated points varied randomly with time. This speckled beam was self-trapped with diffraction being eliminated; the beam envelope maintained a constant diameter throughout propagation ${ }^{13}$. However, that previous experiment served only as a 'proof of principle' as the rotating diffuser generated partially spatially incoherent light only. The light origi- nated from a laser and was temporally coherent (quasi-monochromatic) at all times.

Here we report self-trapping of a broad-band spatially and temporally incoherent light beam, emerging from an incandescent source; a light bulb. We have used a quartz-tungsten-halogen incandescent bulb to generate the white-light beam. The light is initially sent through a spectral filter to limit the frequency band to $380-720 \mathrm{~nm}$ (the temporal coherence time of the beam is of the order of a few femtoseconds). The light was collimated into a beam, sent through a polarizer to keep one polarization only, and focused onto the input face of an SBN:75 photorefractive crystal $\left(\mathrm{Sr}_{0.75} \mathrm{Ba}_{0.25} \mathrm{Nb}_{2} \mathrm{O}_{6}\right)$. The spectrum of the light incident on the crystal is shown in Fig. 1. Our SBN crystal exhibited photorefractivity to wavelengths between roughly 380 and $520 \mathrm{~nm}$, which gives a normalized bandwidth $\Delta \nu / \nu_{0}$ of roughly 0.3 . In the crystal, the incoherent beam propagates along the crystalline $a$-axis with its polarization parallel to the $c$-axis (extraordinary polarization). We used a lens to image the beam at the input and output faces of the crystal onto a CCD (charge-coupled device) camera. As with photorefractive screening solitons ${ }^{18}$, the magnitude of the nonlinearity is fine-tuned with a uniform background beam by generating a bias level of electrons in the conduction band. For this purpose, we use an ordinarily polarized 488-nm laser beam which was expanded to illuminate the crystal uniformly. Selffocusing occurs with the application to the crystal of an appropriate voltage (magnitude and polarity) which gives rise to a space-charge field that has a large component along the $c$-axis, thus using the $r_{33}=1,022 \mathrm{pm} \mathrm{V}^{-1}$ electro-optic coefficient to create the index change required for self-trapping. An input beam of $14 \mu \mathrm{m}$ (all beam sizes are given here as full-width at half-maximum, FWHM) diffracts to $82 \mu \mathrm{m}$ after $6 \mathrm{~mm}$ of propagation. The large diffraction angle demonstrates the spatial incoherence of the beam. A coherent beam of size $14 \mu \mathrm{m}$ at $380 \mathrm{~nm}$ wavelength would have diffracted to $35.34 \mu \mathrm{m}$, whereas the same-size input beam at $720 \mathrm{~nm}$ would have diffracted to $63.1 \mu \mathrm{m}$. Applying $600 \mathrm{~V}$ between the electrodes separated by $6 \mathrm{~mm}$ results in self-trapping of the beam, which traps to $12 \mu \mathrm{m}$. The total optical power in the white-light input beam is $70.8 \mathrm{nW}$. As this power is spread over $340 \mathrm{~nm}$, the amount of light at the photorefractively sensitive wavelengths is $\sim 25 \mathrm{nW}$. The $488-\mathrm{nm}$ background beam had a power of $400 \mathrm{nW}$. These very low power levels, when translated to the corresponding beam intensities, result in a very long formation time (which is related to the dielectric relaxation time) for the self-trapped beam.

Our experimental results are shown in Figs 2 and 3. Figure 2a shows the profile of a $14-\mu \mathrm{m}$ beam at the input face of the crystal. Figure $2 \mathrm{~b}$ shows the profile of the $82-\mu \mathrm{m}$-wide normally diffracting beam in the absence of nonlinearity (zero voltage). Figure $2 c-1$ shows the temporal evolution of the beam at the output face of the crystal that occurs once the nonlinearity (voltage) is turned on. The beam starts to self-focus by going through a quasi-steady-state regime that is reminiscent of quasi-steady-state photorefractive solitons ${ }^{10}$. Then, the beam breaks up and moves towards the positive $c$-axis and forms the steady-state self-trapped beam as shown by Fig. $2 \mathrm{i}-\mathrm{l}$. The centre of the self-trapped beam of Fig. $2 \mathrm{j}$ has moved a distance of $57 \mu \mathrm{m}$ away from the centre of the initial diffracted beam, towards the $c$-axis. This 'displacement' of the self-trapped beam is closely related to the self-bending effects that all photorefractive solitons experience; it is driven by a diffusion field that is the lowest-order correction to the space-charge field that supports the solitons ${ }^{20}$. The self-trapped beam roughly maintains its structure for at least eight hours, during which the beam fluctuated slightly in shape and drifted slowly towards the $c$-axis. It remained a selftrapped entity and did not break up or diminish for the entire duration of our experiment. Possible reasons for the slow fluctuations in the shape of the self-trapped beam are 'environmental' changes, such as temperature variations or slow drifting in the optical power emitted from the sources, as we have not used any 


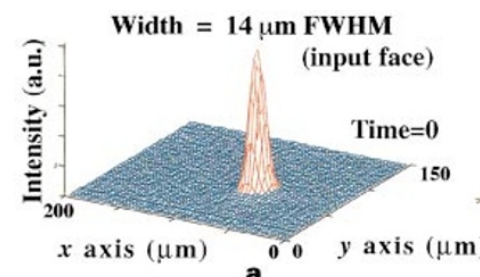

Width $=82 \mu \mathrm{m}$ FWHM (output face)
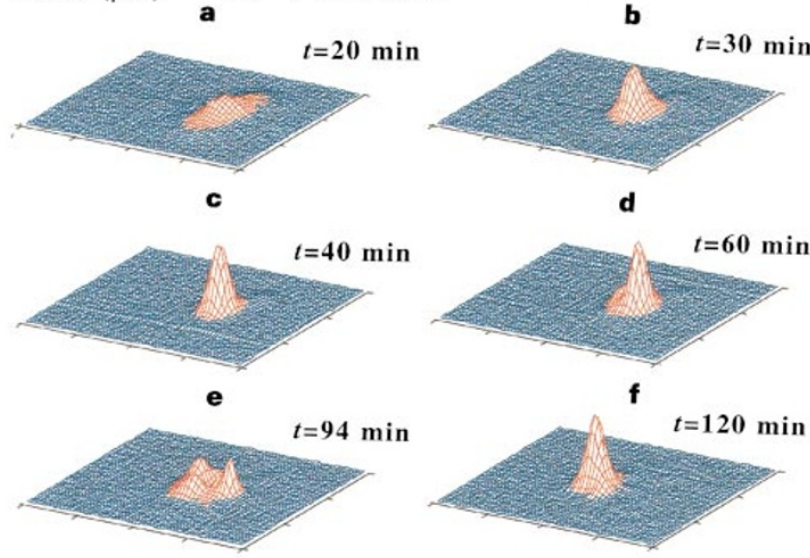

g

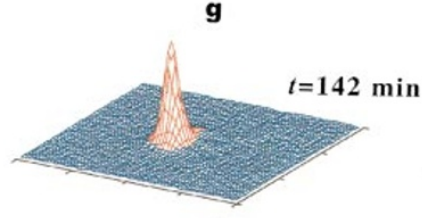

i

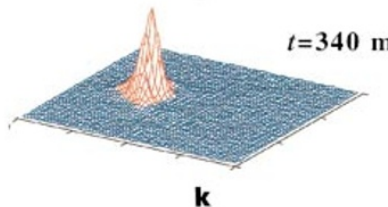

$\mathbf{k}$

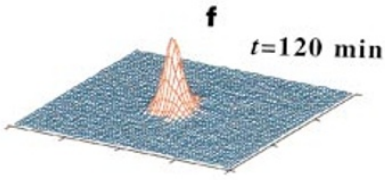

h

Width $=12 \mu \mathrm{m}$

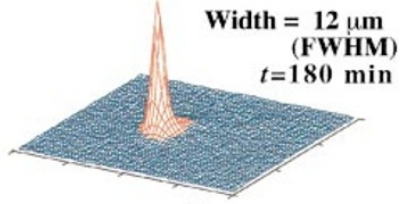

j

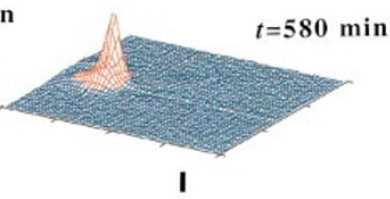

Figure 2 Steady-state self-trapping of a white-light beam. a, Profile of $14-\mu \mathrm{m}$ beam (full-width at half-maximum, FWHM) at input face of crystal; $\mathbf{b}$, profile of $82-\mu \mathrm{m}$ beam at output face of crystal; c-I, temporal evolution of beam at output face of crystal.

special means to isolate our system. We emphasize, however, that the beam remained self-trapped (localized) for as long as we have monitored the experiment. We believe that the small fluctuations are not related to the self-trapping mechanism of the incoherent beam.

Quasi-steady-state trapping was observed even without use of background illumination. Figure $3 \mathrm{a}$ shows the profile of a $26-\mu \mathrm{m}$ beam at the input of the crystal. Figure $3 \mathrm{~b}$ shows the profile of the $100-\mu \mathrm{m}$ normally diffracting beam in the absence of nonlinearity (zero voltage). Figure $3 \mathrm{c}-\mathrm{f}$ shows the temporal evolution of the beam at the output face of the crystal. The beam focuses to a size of 26- $\mu \mathrm{m}$ then breaks up and eventually diminishes.

To our knowledge, this is the first observation of a self-trapped beam from a source that is both temporally and spatially incoherent. The phase across the self-trapped beam is varying in a random manner both in time and in space. Unlike the case of a self-trapped coherent beam, knowing (measuring) the phase at a particular point on the incoherent self-trapped beam cannot provide any phase information, even at very short distances away from that point. Furthermore, at each point on the beam, photons of widely varying frequencies coexist, so the absolute phase of the total optical field varies randomly between each two points separated by a distance of

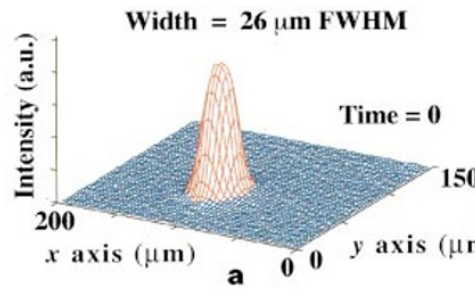

Width $=100 \mu \mathrm{m}$ FWHM

Width $=26 \mu \mathrm{m}$ FWHM
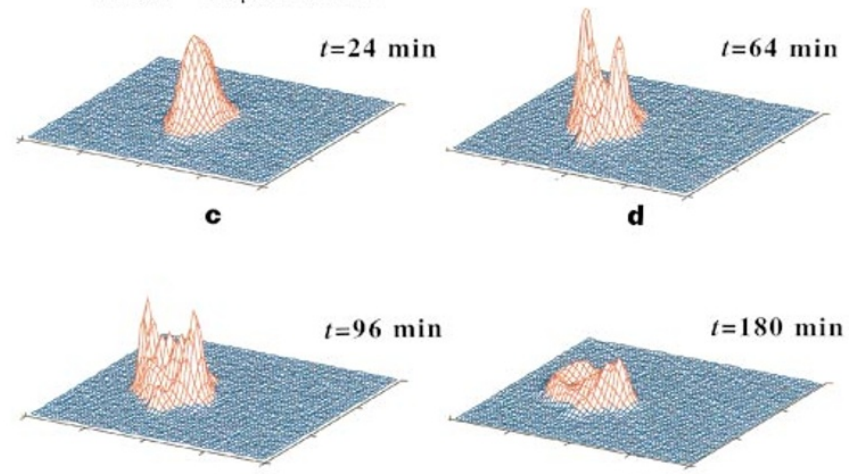

e

$\mathbf{f}$

Figure 3 Quasi-steady-state self-trapping of a white-light beam. a, Profile of 26$\mu \mathrm{m}$ beam at input face of crystal; $\mathbf{b}$, profile of $100-\mu \mathrm{m}$ diffracted beam at output face of crystal; c-f, temporal evolution of beam at output face of crystal.

the order of the optical wavelength. Yet, this incoherent white-light beam indeed self-traps.

Self-trapping of 'white' incoherent light introduces the possibility of using incoherent sources (for example, light-emitting diodes) for optical interconnects, beam steering, and other applications that have been thus far proposed only for (coherent) solitons. On the fundamental level, self-trapping of incoherent light raises many intriguing questions. We believe that the statistics of the self-trapped incoherent beam are affected by the self-trapping: going from the delocalized statistics of the input thermal source (that is, the coherence depends on coordinate difference only) into a state in which the statistics depend also on the absolute coordinate across the self-trapped beam. How are the brightness and entropy, which are related to the coherence properties of the beam, affected by selftrapping? If brightness is improved, it must come at the expense of energy loss, in the form of radiation or absorption. Are self-trapped incoherent beams able to maintain their identities as they undergo collisions with each other? These and other issues are currently under investigation.

Received 4 February; accepted 6 May 1997

1. Chiao, R. Y., Garmire, E. \& Townes, C. H. Self-trapping of optical beams. Phys. Rev. Lett. 13, 479-482 (1964)

2. Hasegawa, A. \& Tappert, F. Transmission of stationary nonlinear optical pulses in dispersive dielectric fibers. Appl. Phys. Lett. 23, 142-144 (1973).

3. Mollenauer, L. F., Stolen, R. H. \& Gordon, J. P. Experimental observation of picosecond pulse narrowing and solitons in optical fibers. Phys. Rev. Lett. 45, 1095-1098 (1980).

4. Snyder, A. W., Mitchell, D. J., Poladian, L. \& Ladouceur, F. Self-induced optical fibers. Opt. Lett. 16, 21-23 (1991).

5. Zabusky, N. J. \& Kruskal, M. D. Interaction of "solitons" in a collisionless plasma and the recurrence of initial states. Phys. Rev. Lett. 15, 240-243 (1965).

6. Bjorkholm, J. E. \& Ashkin, A. Self-focusing and self-trapping of light in sodium vapor. Phys. Rev. Lett. 32, 129-132 (1974).

7. Barthelemy, A., Maneuf, S. \& Froehly, C. Propagation soliton et auto-confinement de faisceaux laser par non linearite optique de kerr. Opt. Commun. 55, 201-206 (1985).

8. Aitchison, J. S. et al. Observation of spatial optical solitons in a nonlinear glass waveguide. Opt. Lett. 15, 471-473 (1990).

9. Segev, M., Crosignani, B., Yariv, A. \& Fischer, B. Spatial solitons in photorefractive media. Phys. Rev. Lett. 68, 923-926 (1992).

10. Duree, G. et al. Observation of self-trapping of an optical beam due to the photorefractive effect. Phys. Rev. Lett. 71, 533-536 (1993). 
11. Torruellas, W. E. et al. Observation of two-dimensional spatial solitary waves in quadratic media. Phys. Rev. Lett. 74, 5036-5039 (1995).

12. Tikhonenko, V., Christou, J. \& Luther-Davies, B. Three dimensional bright spatial soliton collision and fusion in a saturable nonlinear medium. Phys. Rev. Lett. 76, 2698-2701 (1996).

13. Mitchell, M., Chen, Z., Shih, M. \& Segev, M. Self-trapping of partially spatially-incoherent light. Phys. Rev. Lett. 77, 490-493 (1996).

14. Segev, M., Valley, G. C., Crosignani, B., DiPorto, P. \& Yariv, A. Steady state spatial screening-solitons in photorefractive media with external applied field. Phys. Rev. Lett. 73, 3211-3214 (1994).

15. Christodoulides, D. N. \& Carvalho, M. I. Bright, dark and gray spatial soliton states in photorefractive mdeia. J. Opt. Soc. Am. B 12, 1628-1633 (1995).

16. Segev, M., Shih, M. \& Valley, G. C. Photorefractive screening solitons of low and high intensity. J. Opt. Soc. Am. B 13, 706-718 (1996).

17. Iturbe-Castillo, M. D., Marquez-Aguilar, P. A., Sanchez-Mondragon, J. J., Stepanov, S. \& Vysloukh, V. Spatial solitons in photorefractive $\mathrm{Bi}_{12} \mathrm{TiO}_{20}$ with drift mechanism of nonlinearity. Appl. Phys. Lett. 64 408-410 (1994)

18. Shih, M. et al. Observation of two-dimensional steady-state photorefractive screening-solitons. Electron. Lett. 31, 826-827 (1995); Two-dimensional steady-state photorefractive screening solitons. Opt. Lett. 21, 324-326 (1996)

19. Goodman, J. Statistical Optics (Wiley, New York, 1985).

20. Singh, S. R. \& Christodoulides, D. N. Evolution of spatial optical solitons in biased photorefractive media under steady state conditions. Opt. Commun. 118, 569-576 (1995).

Acknowledgements. This work was supported by the US Army Research Office and the US National Science Foundation.

Correspondence should be addressed to M.S. (e-mail: segev@ee.princeton.edu).

\section{Crystallization of hard-sphere colloids in microgravity}

\section{Jixiang Zhu*, Min Li $\dagger$, R. Rogers $\ddagger$, W. Meyer $\ddagger$, R. H. Ottewill $₫$, STS-73 Space Shuttle Crew\|, W. B. Russel $\dagger \&$ P. M. Chaikin ${ }^{\star}$}

* Department of Physics, $†$ Department of Chemical Engineering, Princeton University, Princeton, New Jersey 08544, USA

$\ddagger$ NASA Lewis Research Center, Cleveland, Ohio 44136-3191, USA

$\$$ School of Chemistry, University of Bristol, Bristol BS8 1TS, UK

II Johnson Space Flight Center, Houston, Texas 77058, USA

The structure of, and transitions between, liquids, crystals and glasses have commonly been studied with the hard-sphere model $^{1-5}$, in which the atoms are modelled as spheres that interact only through an infinite repulsion on contact. Suspensions of uniform colloidal polymer particles are good approximations to hard spheres $^{6-11}$, and so provide an experimental model system for investigating hard-sphere phases. They display a crystallization transition driven by entropy alone. Because the particles are much larger than atoms, and the crystals are weakly bound, gravity plays a significant role in the formation and structure of these colloidal crystals. Here we report the results of microgravity experiments performed on the Space Shuttle Columbia to elucidate the effects of gravity on colloidal crystallization. Whereas in normal gravity colloidal crystals grown just above the volume fraction at melting show a mixture of random stacking of hexagonally close-packed planes (r.h.c.p.) and face-centred cubic (f.c.c.) packing if allowed time to settle ${ }^{7,8}$, those in microgravity exhibit the r.h.c.p. structure alone, suggesting that the f.c.c. component may be induced by gravity-induced stresses. We also see dendritic growth instabilities that are not evident in normal gravity, presumably because they are disrupted by shear-induced stresses as the crystals settle under gravity. Finally, glassy samples at high volume fraction which fail to crystallize after more than a year on Earth crystallize fully in less than two weeks in microgravity. Clearly gravity masks or alters some of the intrinsic aspects of colloidal crystallization.

The thermodynamic phase diagram for hard spheres as obtained by computer simulations is: $\phi_{\text {liquid }}<\phi_{\text {freeze }}=0.494<\phi_{\text {coexist }}<\phi_{\text {melt }}$ $=0.545<\phi_{\text {crystal }}<0.74$ (refs 1-4), where $\phi$ is the volume fraction; the simulations also find reduced diffusion and a metastable glass phase for $\phi>0.58$. For typical hard-sphere colloidal systems on Earth the gravitational length $h$ is about $1-30 \mu \mathrm{m}(m g h=k T$, where $m$ is the buoyant mass of a particle, $g$ is the acceleration due to gravity, $k$ is the Boltzmann's constant and $T$ is temperature), below crystallite sizes, and much less than the sample sizes (of the order of centimetres). So it is not clear what effects sedimentation, concentration gradients and gravitational stresses have on the kinetics and thermodynamics of the crystals ${ }^{12}$.

The particles consist of uniform poly(methylmethacrylate) (PMMA) spheres, 508 or $518 \mathrm{~nm}$ in diameter $\left(\equiv 2 a_{0}\right)$, polydispersity $\sim 5 \%$, with a thin $(10-\mathrm{nm})$ grafted layer of poly(hydroxystearic acid) to prevent aggregation ${ }^{11}$. The particles are suspended in an index of refraction (1.51) matching mixture of decalin and tetralin. Using a stirring bar in the sample cells, the samples were mixed (shear melted) by the astronauts on day 2 of the flight. The static laser light scattering (wavelength, $\lambda=791 \mathrm{~nm}$ ) was obtained with a $50-\mu \mathrm{m}$ beam focused through a cylindrical sample and lens on a translucent screen and recorded with a video camera. The sample was translated to obtain data from many independent crystallites.

Crystal close packing can be obtained by stacking hexagonal planes of spheres. With a first layer as A, there are two equivalent placements $B$ and $C$ of the second layer above the interstitial sites of the first. If the stacking continues, the f.c.c. lattice is the arrangement $\mathrm{ABCABC...,} \mathrm{hexagonal} \mathrm{close-packed} \mathrm{(h.c.p.)} \mathrm{is} \mathrm{ABABAB} . .$, but any arrangement has the same volume fraction, $\phi=0.7404$. The random arrangement $\mathrm{ABACBACBCA...} \mathrm{with} \mathrm{no} \mathrm{repeating} \mathrm{sequence}$
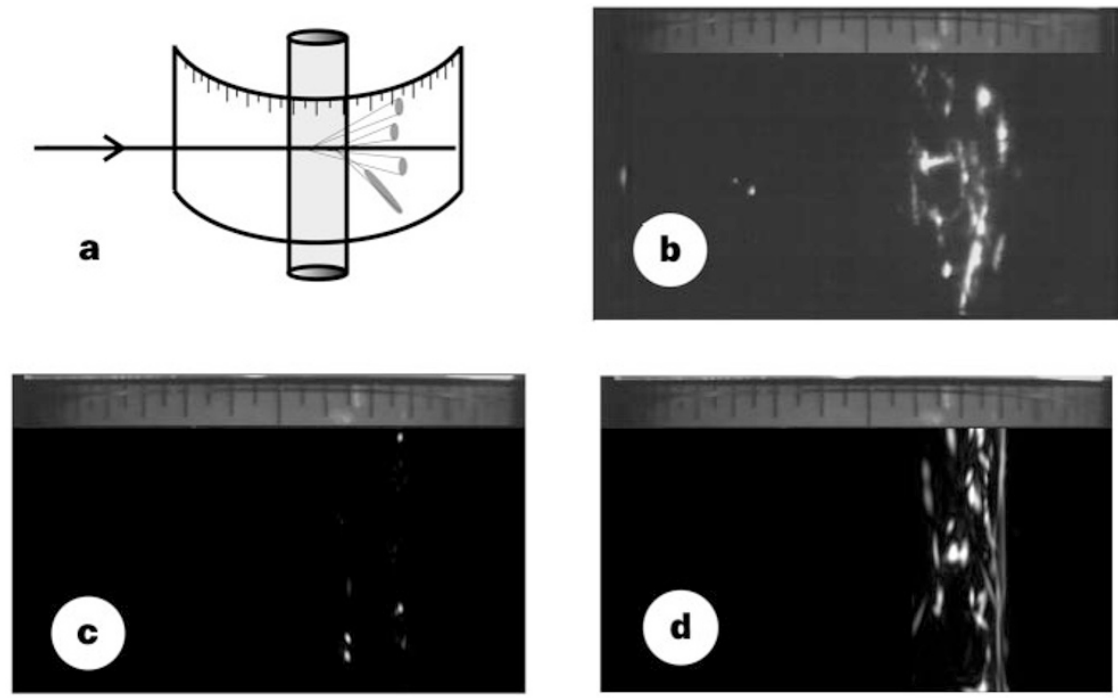

Figure $1 \mathbf{a}$, Bragg scattering geometry. A laser beam incident on a cylindrical sample is scattered onto a cylindrical screen. b, Bragg scattering from a sample with $2 a_{0}=518 \mathrm{~nm}$ and $\phi=0.537$. Note the dominance of streaks rather than spots, indicating Bragg rods and a two-dimensional structure as might be expected for a random hexagonal close-packed (r.h.c.p.) structure. c, d, Computer-generated scattering pattern for f.c.c. (c) and r.h.c.p. (d) structures. 